\title{
Mitochondrial peroxidase TPx-2 is not essential in the blood and insect stages of Plasmodium berghei
}

Hirono Masuda-Suganuma, Miho Usui, Shinya Fukumoto, Noboru Inoue and Shin-ichiro Kawazu*

\begin{abstract}
Background: Malaria parasites actively proliferate in the body of their vertebrate and insect hosts, and are subjected to the toxic effects of reactive oxygen species. The antioxidant defenses of malaria parasites are considered to play essential roles in their survival and are thus considered promising targets for intervention. We sought to identify the cellular function of thioredoxin peroxidase-2 (TPX-2), which is expressed in the mitochondria, by disrupting the TPx-2 gene (pbtpx-2) of the rodent malaria parasite Plasmodium berghei.
\end{abstract}

Findings: In three independent experiments, two disruptant populations (TPx-2 KO) and three wild-type parasite populations with pyrimethamine resistance (dhfr-ts/mt at the DHFR-TS locus) and intact pbtpx-2 (TPx-2 WT) were obtained and cloned. Null expression of TPx-2 in the KO population was confirmed by RT-PCR and Western blot analyses. The TPx-2 KO parasite developed normally in mouse erythrocytes and multiplied at a rate similar to that of the TPx-2 WT parasite during the experimental period. The peak period of gametocytemia was delayed by 1 day in the TPx-2 KO compared with that of the TPx-2 WT and the parent parasite, however, the highest gametocyte number was comparable. The number of midgut oocysts in the TPX-2 KO at 14 days post feeding was comparable to that of the TPX-2 WT.

Conclusions: The present finding suggests that mitochondrial Prx TPx-2 is not essential for asexual and the insect stage development of the malaria parasite.

Keywords: Peroxiredoxin, Plasmodium berghei, Thioredoxin peroxidase

\section{Findings Introduction}

Malaria is a disease caused by infection with protozoan parasites of the genus Plasmodium and transmitted by Anopheles mosquitoes. Almost half of the world's population lives with a serious risk of contacting malaria, and nearly 1 million people died from the disease in 2010 [1]. Despite years of intensive research, an effective vaccine is still not available, and the parasite displays increasing resistance towards the commonly used anti-malarial drugs. To combat malaria, a better understanding of the basic biology of the parasite is needed, especially with regard to the mechanisms of adaptation to environmental conditions.

\footnotetext{
* Correspondence: skawazu@obihiro.ac.jp

National Research Center for Protozoan Diseases, Obihiro University of Agriculture and Veterinary Medicine, Inada, Obihiro 080-8555, Japan
}

As Plasmodium spp. actively proliferate in the erythrocytes of their vertebrate hosts, large quantities of reactive oxygen species (ROS), which damage biological macromolecules, are generated in the cell during parasite development $[2,3]$. One of the major sources of ROS in the parasite cell is heme, which is produced as a byproduct of hemoglobin digestion for amino acid procurement [4]. In addition, the parasite possesses a mitochondrion with a functional electron transport chain. During respiration, $\mathrm{ROS}$ are generated that need to be removed. ROS also emerge in the cell when the organism is exposed to a variety of stress conditions such as the action of the host immune system [5]. Since Plasmodium spp. are highly susceptible to oxidative stresses, their antioxidant defenses are considered to play essential roles in their development, and they are thus expected to be potential targets for chemotherapy [6]. 
Malaria parasites possess six peroxidases localized in the cytoplasm, mitochondrion, apicoplast and nucleus: a 1-Cys Prx, two typical 2-Cys Prxs, a 1-Cys antioxidant protein (AOP), a Prx family with unusual biochemical characteristics and a glutathione (GSH) peroxidase-like thioredoxin peroxidase $\left(\mathrm{TPx}_{\mathrm{Gl}}\right)$ [7-9]. Since malaria parasites do not possess catalase and genuine $\mathrm{GSH}$ peroxidase in their genome, it is considered that GSH itself is the major redox buffer for transient $\mathrm{H}_{2} \mathrm{O}_{2}$ exposure and the basal peroxide flux in the cell is dealt with by the Trx system [6]. It has recently been reported that disruption of the gene for the cytosolic 2-Cys Prx (TPx-1) in Plasmodium renders parasites hypersensitive to ROS and reactive nitrogen species, although it did not affect parasite growth under normal in vitro and in vivo asexual development [10]. Thus far, however, the functions of the Prx family in the organelle are unclear. In the present study, the gene coding for TPx2 , which is expressed in the mitochondrion of the rodent malaria parasite $P$. berghei (Additional file 1: Figure S1), was disrupted and phenotypes of the disruptant were observed in experimental infections in mice and mosquitoes in order to investigate unidentified cellular function of the Prx family in malaria parasites.

\section{Methods}

The $P$. berghei ANKA strain was obtained from the Armed Forces Research Institute of Medical Sciences, Thailand. The parasite was maintained by mosquito transmission in Anopheles stephensi interspersed by a maximum of two serial passages in BALB/c mice (Clea Tokyo, Japan).

For disruption of the pbtpx-2,5/ and 3/ portions of the gene were amplified by polymerase chain reaction (PCR) and cloned into the targeting vector pMD204 [11], which was supplied by the Malaria Research and Reference Reagent Resource Center; MR4/ATCC, Manassas, VA, USA. Each fragment contained part of the coding sequence and flanking region and was amplified with sequence-specific primers and parasite genomic DNA. The primers used for the $5^{\prime}$ fragment were $5^{\prime}$-GGG CCC CTA TCT GAG TAA TAT CTC ATA TCT $\overline{\mathrm{CC}-3^{\prime}}$ and 5'-GAG CTC CAT GAG AAA ACG ATC TCA CTG ATC-3' (Apa I and Xho I sites are underlined). The primers used for the $3^{\prime}$ fragment were $5^{\prime}$-GAA TTC GTT TAG ACC CAA TAA TGA GGC-3 ${ }^{\prime}$ and $5^{\prime}-\underline{\text { CTG }}$ CAG GTT TTT CAC ACA CAG GAG TTA C-3’ $\overline{(E c o}$ RI and Pst I sites are underlined). The primers were designed on the basis of sequences in the P. berghei genome database provided by the Plasmodium Genome Resource [PlasmoDB: PBANKA_143080]. PCR products were purified and cloned into the upstream or downstream of the pyrimethamine-resistant form of the $P$. berghei dihydrofolate reductase-thymidylate synthase gene (dhfr-ts), which was used as a selectable marker.
For gene targeting experiments, the plasmid was digested with Apa I and Pst I to separate the linear targeting construct from the plasmid backbone.

Transfection was performed according to the protocol of Janse et al. [12]. To transfect the knock out construct into P. berghei with Nucleofector ${ }^{\circledR}$ (Amaxa, Germany), mature schizonts were prepared. To collect the parasites, ICR mice (Clea Japan) were infected with $P$. berghei by intraperitoneal (i.p.) injection of $5 \times 10^{6}$ parasitized erythrocytes per animal. The schizont pellet was resuspended in $0.1 \mathrm{ml}$ Nucleofector ${ }^{\circledR}$ working solution containing $9 \mu \mathrm{g}$ linearized targeting vector, then transferred to electroporation cuvettes for transfection at the U-33 setting. After transfection, $50 \mu \mathrm{l}$ of complete culture medium was added to the mixture and was immediately inoculated into a mouse per transfection by intravenous (i.v.) injection. The mice were provided with drinking water containing pyrimethamine $(0.7 \mathrm{mg} / \mathrm{ml}) 1$ day after infection with transfected parasites. When parasitemia had reached $1-2 \%$, the parasite population in each mouse was examined by allele-specific PCR prior to cloning the parasite population with the wild-type and disruptant allele by limiting dilution. Both parasite genomes were determined by PCR and Southern blot analyses. Null-phenotype for TPx-2 expression was confirmed by reverse transcriptase PCR (RT-PCR) and Western blotting.

Five-week-old female BALB/c mice (CLEA Japan, Japan) were infected with $P$. berghei $\left(10^{6}\right.$ parasitized erythrocytes per mouse) by i.p. injection. Parasitemia and gametocytemia were determined by microscopic examination of Giemsa-stained thin blood films. Gametocytes were distinguished by size and coloration. The animal experiments in this study were carried out in compliance with the Guide for Animal Experimentation at Obihiro University of Agriculture and Veterinary Medicine (permission numbers: 21-56 and 22-48).

Six-week-old male BALB/c mice (CLEA Japan, Japan) were infected with $P$. berghei by i.p. injection of the parasite, which had been stored as frozen stock at $-80^{\circ} \mathrm{C}$. Parasitemia of each mouse was monitored daily by light microscopic observation of Giemsa-stained thin blood smears. A. stephensi mosquitoes were maintained on $10 \%$ sugar solution at $27^{\circ} \mathrm{C}$ and $80 \%$ relative humidity under a $12 \mathrm{hr}$ light/dark cycle. When the number of microgametocytes that could exflagellate in vitro [13] had reached 2030 per $1 \times 10^{5}$ erythrocytes, the mosquitoes were fed on the mice for $2 \mathrm{~h}$ at $19^{\circ} \mathrm{C}$. The parasite-infected mosquitoes (100-200 mosquitoes per group) were maintained at $19^{\circ} \mathrm{C}$ with $10 \%$ sugar solution.

At 14 days post-feeding, mosquitoes that had been infected with parasite populations were dissected and their midguts were isolated. The midguts were stained using the improved technique [14]. Briefly, the midguts 
were stained with $0.5 \%$ mercurochrome (Sigma Aldrich Japan, Japan) in water at room temperature for $10 \mathrm{~min}$ and washed in PBS for $30 \mathrm{~min}$. Next, stained midguts were fixed with $10 \%$ formalin (Wako Pure Chemical, Japan) for $24 \mathrm{~h}$ and opened along the median line. The midgut specimens were observed, and the numbers of red-stained oocysts were counted using light microscopy (x 100 or x 200).

Differences were evaluated with the Student's $t$-test (mouse infection) or the Mann-Whitney test (mosquito infection). $P<0.05$ was considered statistically significant.

\section{Results and discussion}

Merozoites in segmented schizonts were transfected with the targeting construct by nucleofection and were subsequently inoculated into naïve mice. Integration of the construct into the pbtpx-2 locus by homologous recombination resulted in disruption of the single-copy gene and insertion of a selectable marker, the dhfr-ts with a pyrimethamine-resistance mutation (dhfr-ts/mt), at this locus (Figure 1A). Parasites with pbtpx-2 disruption were selected by treatment with pyrimethamine. The allele-specific PCR analysis showed that parasites selected with pyrimethamine were a mixture of wildtype parasites and $p b t p x-2$ disruptants (data not shown). Parasite populations were separated into two groups by limiting dilution and subsequent inoculation into 37 rats. In three independent electroporation experiments, two disruptant (TPx-2 KO) and three wild-type parasite clones with pyrimethamine resistance (dhfr-ts/mt at the $d h f r$-ts locus) and intact pbtpx-2 (TPx-2 WT) were established. In the first experiment, three TPx-2 WT clones were obtained. Each TPx-2 KO clone was obtained from the second and third experiments, respectively. Southern blot analysis confirmed the replacement of the pbtpx-2 locus by the targeting construct in the TPx-2 KO genome (Figure 1B). Null expression of TPx-2 in the KO clone was confirmed by RT-PCR and Western blot analyses (Figure 2). The dhfr-ts locus of the TPx-2 WT clone was amplified by PCR and sequenced, and replacement with the pyrimethamineresistance mutation was confirmed (data not shown).

In vivo asexual growth was compared in TPx-2 $\mathrm{KO}$ and TPx-2 WT clones. For these experiments, BALB/c mice were infected with the parasite clones by i.p. injection, and the course of parasitemia was determined. The TPx-2 WT clone showed equal levels of development and multiplication within erythrocytes, similar to the parent strain (WT) (data not shown), and they showed a high level of parasitemia $(>5 \%) 4$ days after infection (Figure 3A). The course of parasitemia observed in TPx-2 KO1-infected mice was similar to that of TPx-2 WT-infected mice. This phenotype was confirmed in TPx-2 KO2, which was obtained from an independent electroporation experiment (Figure 3A). There was no difference in the morphology of parasite cells between two TPx-2 KO, TPx-2 WT, and WT clones (data not shown). To evaluate the effect of pbtpx-2 disruption on development of sexual-stage parasites, the numbers of

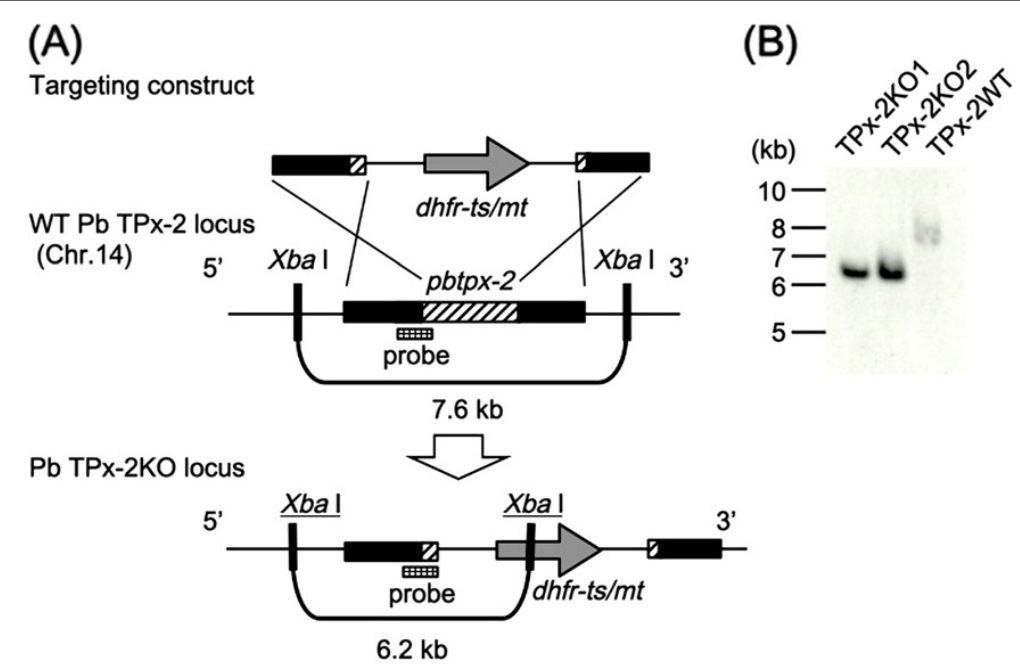

Figure 1 Targeted disruption of the tpx-2 gene in P. berghei. (A) The targeting construct, composed of the $5^{\prime}$ and $3^{\prime}$ regions (filled boxes) and partial open reading frame (hatched boxes) of the pbtpx-2 and the pyrimethamine-resistant variant of dhfr-ts of $P$. berghei (dhfr-ts/mt) as a selectable marker (gray arrow), was integrated into the wild-type (WT) PbTPx-2 locus by double-crossover homologous recombination. Recombination disrupts pbtpx-2 and creates the locus containing dhfr-ts/mt (PbTPx-2 KO locus), which confers pyrimethamine resistance to disruptants. (B) Southern blot analysis of genomic DNA samples from the wild-type parasite populations with pyrimethamine resistance (dhfr-ts/ $m t$ at the dhfr-ts locus) (TPX-2 WT), and two pbtpx-2 disruptant populations (TPX-2 KO). DNA samples were digested with Xba I, separated on 0.7\% agarose gels, transferred to nylon membranes, and hybridized with a probe. The single 6.2-kb band in the TPX-2 KO populations indicates pbtpx-2 disruption, whereas the single 7.6-kb band in the TPx-2 WT populations indicates an intact pbtpx-2 locus. 


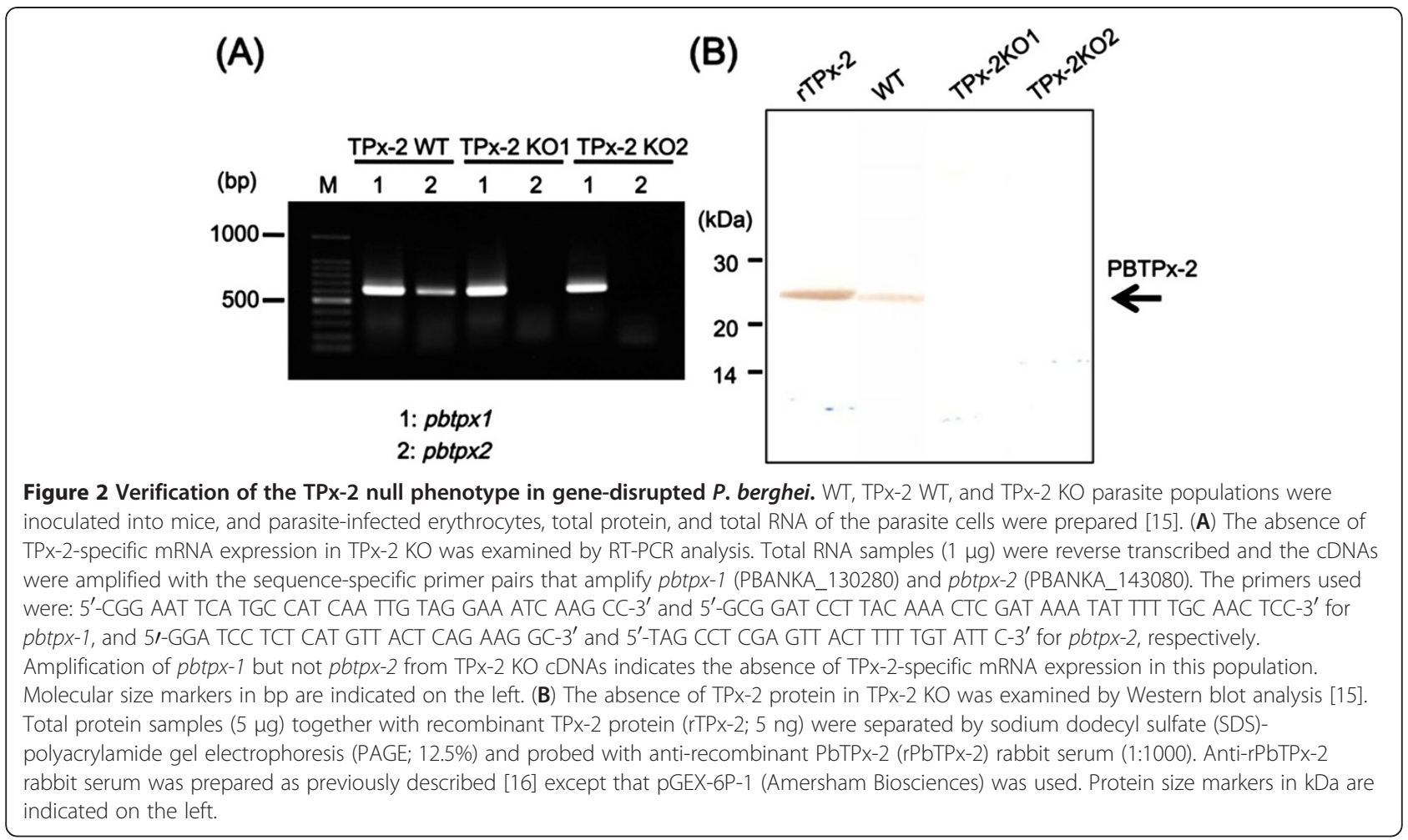

gametocytes in parasite-infected blood were counted and compared among TPx-2 KO1-2, TPx-2 WT and WT (Figure 3B). In TPx-2 WT-infected mice, gametocytes were observed from 2 days after infection; they increased in number with parasitemia progression and peaked 4 days after infection. The course of gametocytemia observed in TPx-2 WT-infected mice was similar to that of WT-infected mice. Gametocytemia, recorded as the number of gametocytes $/ 10^{4}$ erythrocytes in TPx-2 WT and WT-infected mice at 4 day of infection, was $19.0 \pm 12.0$ and $26.0 \pm 10.0$, respectively $(P>0.05)$. In TPx-2 KO1-2-infected mice, gametocytes were also observed from 2 days after infection but peaked 5 days after infection. The peak of gametocytemia in TPx-2
KO-infected mice was thus delayed by 1 day compared with that of Prx WT- and WT-infected mice. The difference between WT and TPx-2 KO populations in the number of days required for having the peak gametocyte number was significant $(P<0.05)$. Gametocytemia, recorded in TPx-2 KO1-2-infected mice as the number of gametocytes $/ 10^{4}$ erythrocytes on day 5 of infection, was $28.0 \pm 9.0$ and $24.0 \pm 8.0$, respectively. The peak number of gametocytes in TPx-2 KO1-2-infected mice was thus comparable to that of TPx-2 WT-infected mice $(P>0.05)$.

To investigate the effect of pbtpx-2 disruption in the insect stage, mosquitoes were fed on parasite infected BALB/c mice that had been infected with TPx-2 WT
(A)

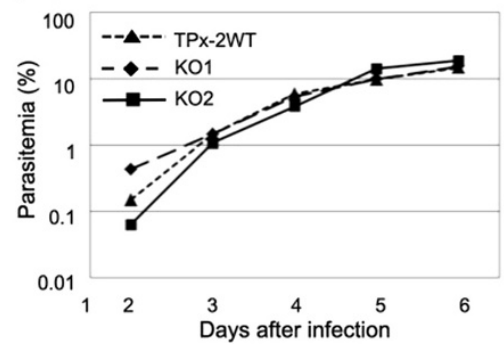

(B)



Figure 3 Infection of mice with tpx-2-disrupted P. berghei. Parasite populations, including WT, TPx-2 WT and two TPx-2 KO populations (KO1 and KO2), were inoculated into mice, and the courses of parasite development in erythrocytes were observed. (A) Changes in parasitemia 6 days after infection (three mice per group). (B) Changes in gametocytemia 6 days after infection (four mice per group). Gametocyte numbers were shown as gametocytes $/ 10^{4}$ erythrocytes. Data show mean values of parasitemia percentage (A) and gametocyte number (B). *Significant difference in the days required for having the highest gametocyte number between WT and TPx-2 KO populations $(P<0.05$; Student's $t$-test). 
and two TPx-2 KO populations. At 14 days post-feeding, the mosquitoes were dissected, and oocyst numbers in the midgut were counted. In this experiment, the number of midgut oocysts in TPx-2 KO populations at 14 days post-feeding was comparable to those of the TPx-2 WT $(P>0.05)$ (Figure 4$)$. There was no significant difference in the developmental stages of oocysts between TPx-2 KO and TPx-2 WT populations (data not shown), and this finding suggested that TPx-2 $\mathrm{KO}$ oocysts could develop similarly to those of TPx-2 WT in the midgut.

The results presented here suggest that $p b t p x-2$ disruption does not affect asexual intraerythrocytic growth of parasites. The present findings might be contradicted by the facts that the mitochondria of the rodent malaria parasite, $P$. yoelii, possess an active respiratory chain [17] and the mitochondrial peroxide-detoxifying capacity is likely to be provided by Prx [18]. It has also been reported in other organisms that a mitochondrial Trx system plays pivotal roles in defense against oxidative stress generated in the organelle [19].

In P. falciparum, it had been believed for a long time that the parasite electron transport chain was not a source of ATP, but was essential in the parasite for maintenance of mitochondrial membrane potential, as indicated by their hypersensitivity to proguanil, a drug that collapsed the membrane potential in the presence of an electron transport inhibitor [20,21]. However, it has been shown recently that metabolism in $P$. falciparum grown in human patients is affected by varied oxygen and substrate levels and by host-parasite interactions. As a consequence, the parasite seems to induce expression of genes associated

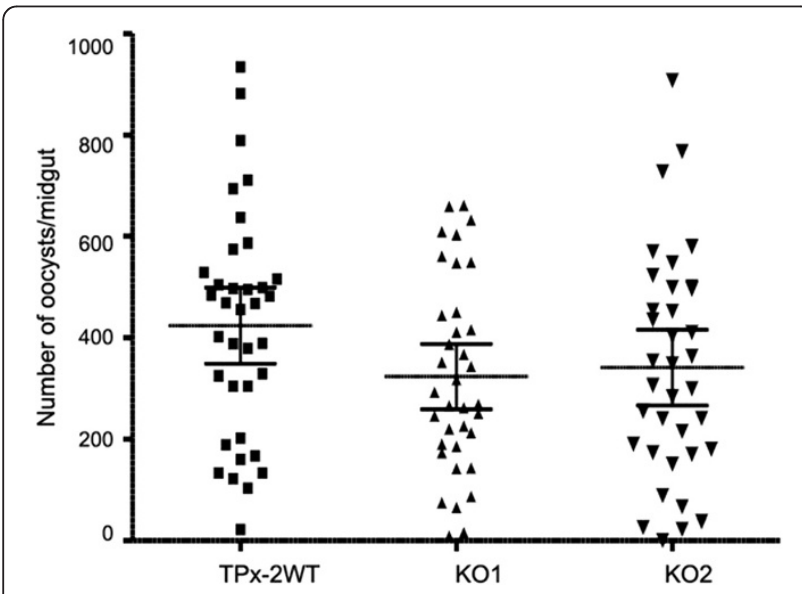

Figure 4 Number of oocysts per midgut. Data were obtained from TPX-2 WT and two TPX-2 KO populations (KO1 and KO2). The line indicates the $95 \%$ confidence interval of the mean. The oocyst formation rate of the TPX-2 KO populations was the same as for the TPX-2 WT population ( $P>0.05$; Mann-Whitney test). The number of mosquitoes dissected was 36 for each experimental group. The figure shows representative results of three independent experiments. with oxidative phosphorylation as a response against starvation status [22]. The parasite may require TPx-2 for development under such stressful conditions. The phenotype of TPx-2 KO during asexual growth under low glucose supply would be an interesting subject of investigation.

Plasmodium mitochondria are morphologically different between the sexual and asexual blood stages [23]. Asexual stage parasites have a tubular-like cristate mitochondrion. In contrast, the mature gametocyte stage has multiple (4-8) mitochondria with greater numbers of cristate structures. The differences suggest that the gametocytes might have a higher demand for energy transduction and are also more metabolically active than the asexual stages [23]. Although the contribution of TPx-2 in gametocyte development remains unknown, the phenotype of TPx-2 $\mathrm{KO}$ in the gametocyte stage is thus an interesting subject for further investigation.

The results from phenotype observation in the insect stage suggest that $p b t p x-2$ disruption does not affect gamete fertilization, ookinete formation and transformation of the ookinetes to oocysts, which requires ookinete invasion of epithelial cells and attachment to the basal lamina of the mosquito's midgut. However, it has been reported that ookinetes are subject to oxidative stress during their invasion of midgut epithelial cells [24]. In addition, it is postulated that ROS is produced in the motile ookinete stage, when oxygen metabolism in the cell may be elevated. The expression profiles of the molecules of the Prx family in the TPx-2 KO ookinete would also be worth investigating.

\section{Conclusions}

The present finding suggests that mitochondrial Prx TPx-2 is not essential for the asexual and insect developmental stages of the malaria parasite. Under more pronounced stress conditions, specific functions of TPx-2 may become evident. An explanation for the lack of phenotype in the TPx-2 KO parasites in the blood and insect-stages might be due to redundancy in the function of the multiple members of the thioredoxin system [13]. A multiple Prx $\mathrm{KO}$ clone has not yet been produced in $P$. berghei. It will be interesting to develop such $\mathrm{KO}$ clones in the future to clarify the Prx family, which may functionally overlap with TPx-2 in the malaria parasite.

\section{Additional file}

Additional file 1: Figure S1. Alignment of the deduced amino acid sequences of Plasmodium berghei TPx-2 (PbTPx-2), with those of TPx-2 from other Plasmodium species.

\section{Abbreviations}

GSH: Glutathione; Prx: Peroxiredoxin; TPx: Thioredoxin peroxidase; ROS: Reactive oxygen species. 


\section{Competing interests}

The authors have no competing interests.

\section{Authors' contributions}

HMS and MU: designed and performed the experiments and redacted the manuscript. SF, NI and SIK: contributed to the study design and edited the manuscript. All authors read and approved the final manuscript.

\section{Acknowledgments}

We are very grateful to Dr. A. P. Waters of Glasgow University (Glasgow, Scotland) for providing the targeting vector, pMD204, to the Malaria Research and Reference Reagent Resource Center; MR4/ATCC. We are also grateful to Dr. K. Yano of the National Center for Global Health and Medicine (Tokyo, Japan) and Dr. S. Kanzok of Loyola University (Chicago, USA) for kind advice regarding the gene-targeting experiments and mosquito stage experiments, respectively. This work was supported by a Grant-in-Aid for Scientific Research (20390118 and 23390098) from the Japan Society for the Promotion of Science.

Received: 2 July 2012 Accepted: 8 November 2012

Published: 12 November 2012

\section{References}

1. WHO: Summary and Key Points, World Malaria Report 2011. Geneva: WHO; 2011:X-XII.

2. Becker K, Tilley L, Vennerstrom $J$, Roberts D, Rogerson S, Ginsburg H: Oxidative stress in malaria parasite-infected erythrocytes: host-parasite interactions. Int J Parasitol 2004, 34:163-189.

3. Müller S: Redox and antioxidant systems of the malaria parasite Plasmodium falciparum. Mol Microbiol 2004, 53:1291-1305.

4. Ginsburg H, Ward SA, Bray PG: An integrated model of chloroquine action. Parasitol Today 1999, 15:357-360.

5. Postma NS, Mommers EC, Eling WM, Zuidema J: Oxidative stress in malaria; implications for prevention and therapy. Pharm World Sci 1996, 18:121-129.

6. Krauth-Siegel RL, Bauer H, Schirmer RH: Dithiol proteins as guardians of the intracellular redox milieu in parasites: old and new drug targets in trypanosomes and malaria-causing plasmodia. Angew Chem Int Ed Engl 2005, 44:690-715.

7. Nickel C, Rahlfs S, Deponte M, Koncarevic S, Becker K: Thioredoxin networks in the malarial parasite Plasmodium falciparum. Antioxid Redox Signal 2006, 8:1227-1239.

8. Kehr S, Sturm N, Rahlfs S, Przyborski JM, Becker K: Compartmentation of redox metabolism in malaria parasites. PLOS Pathog 2010, 6:e1001242.

9. Richard D, Bartfai R, Volz J, Ralph SA, Muller S, Stunnenberg HG, Cowman AF: A genome-wide chromatin-associated nuclear peroxiredoxin from the malaria parasite Plasmodium falciparum. J Biol Chem 2011, 286:11746-11755.

10. Kawazu S, Komaki-Yasuda K, Oku H, Kano S: Peroxiredoxins in malaria parasites: parasitologic aspects. Parasitol Int 2008, 57:1-7.

11. van Dijk MR, Waters AP, Janse CJ: Stable transfection of malaria parasite blood stages. Science 1995, 268:1358-1362.

12. Janse CJ, Ramesar J, Waters AP: High-efficiency transfection and drug selection of genetically transformed blood stages of the rodent malaria parasite Plasmodium berghei. Nat Protoc 2006, 1:346-356.

13. Cao YM, Tsuboi T, Torii M: Nitric oxide inhibits the development of Plasmodium yoelii gametocytes into gametes. Parasitol Int 1998, 47:157-166.

14. Hall N, Karras M, Rain JD, Carlton JM, Kooij TW, Berriman M, Florens L, Janssen CS, Pain A, Christophides GK, James K, Rutherford K, Harris B, Harris D, Churcher C, Quail MA, Ormond D, Doggett J, Trueman HE, Mendoza J, Bidwell SL, Rajandream MA, Carucci DJ, Yates JR 3rd, Kafatos FC, Janse CJ, Barrell B, Turner CM, Waters AP, Sinden RE: A comprehensive survey of the Plasmodium life cycle by genomic, transcriptomic, and proteomic analyses. Science 2005, 307:82-86.

15. Yano K, Komaki-Yasuda K, Tsuboi T, Torii M, Kano S, Kawazu S: 2-Cys Peroxiredoxin TPx-1 is involved in gametocyte development in Plasmodium berghei. Mol Biochem Parasitol 2006, 148:44-51.

16. Yano K, Komaki-Yasuda K, Kobayashi T, Takemae H, Kita K, Kano S, Kawazu S: Expression of mRNAs and proteins for peroxiredoxins in Plasmodium falciparum erythrocytic stage. Parasitol Int 2005, 54:35-41.

17. Uyemura SA, Luo S, Vieira M, Moreno SNJ, Docampo R: Oxidative phosphorylation and rotenone-insensitive malate- and $\mathrm{NADH}$-quinone oxidoreductases in Plasmodium yoelii yoelii mitochondria in situ. J Biol Chem 2004, 279:385-393.

18. Boucher IW, McMillan PJ, Gabrielsen M, Akerman SE, Brannigan JA, Schnick C, Brzozowski AM, Wilkinson AJ, Müller S: Structural and biochemical characterization of a mitochondrial peroxiredoxin from Plasmodium falciparum. Mol Microbiol 2006, 61:948-959.

19. Wheeler GL, Grant CM: Regulation of redox homeostasis in the yeast Saccharomyces cerevisiae. Physiol Plant 2004, 120:12-20.

20. Painter HJ, Morrisey JM, Mather MW, Vaidya AB: Specific role of mitochondrial electron transport in blood-stage Plasmodium falciparum. Nature 2007, 446:88-91.

21. van Dooren GG, Stimmler LM, McFadden GI: Metabolic maps and functions of the Plasmodium mitochondrion. FEMS Microbiol Rev 2006, 30:596-630

22. Daily JP, Scanfeld D, Pochet N, Le Roch K, Plouffe D, Kamal M, Sarr O, Mboup S, Ndir O, Wypij D, Levasseur K, Thomas E, Tamayo P, Dong C, Zhou Y, Lander ES, Ndiaye D, Wirth D, Winzeler EA, Mesirov JP, Regev A: Distinct physiological states of Plasmodium falciparum in malaria-infected patients. Nature 2007, 450:1091-1095.

23. Krungkrai J: The multiple roles of the mitochondrion of the malaria parasite. Parasitology 2004, 129:511-524.

24. Han YS, Thompson J, Kafatos FC, Barillas-Mury C: Molecular interactions between Anopheles stephensi midgut cells and Plasmodium berghei: the time bomb theory of ookinete invasion of mosquitoes. EMBO J 2000, 19:6030-6040.

doi:10.1186/1756-3305-5-252

Cite this article as: Masuda-Suganuma et al:: Mitochondrial peroxidase TPX-2 is not essential in the blood and insect stages of Plasmodium berghei. Parasites \& Vectors 2012 5:252.

\section{Submit your next manuscript to BioMed Central and take full advantage of:}

- Convenient online submission

- Thorough peer review

- No space constraints or color figure charges

- Immediate publication on acceptance

- Inclusion in PubMed, CAS, Scopus and Google Scholar

- Research which is freely available for redistribution

Submit your manuscript at www.biomedcentral.com/submit
C BioMed Central 